\title{
Meeting report: 10th International Mouse Genome Conference
}

\author{
Sally A. Camper, Miriam H. Meisler \\ Department of Human Genetics, University of Michigan, Ann Arbor, Michigan 48109-0618, USA
}

Received: 27 February 1997 / Accepted: 3 March 1997

Ten years after hosting the First International Mammalian Genome Conference in Paris in 1986, Dr. Jean-Louis Guénet presided over the Tenth Conference at the Pasteur Institute, October 7-10, 1996. The 1986 conference was a satellite to the Human Gene Mapping Workshop and had approximately 50 attendees. The 1996 meeting was attended by 300 scientists from around the world. In the interim, the number of mapped loci in the mouse increased from 1,000 to over 20,000. The contributions of Dr. Guénet to this decade of progress include more than 60 published gene mapping reports, the development of interspecific backcrosses for highresolution genetic mapping [1], and ongoing investigations of mouse models of human neuromuscular disorders.

This Conference was dedicated to the memory of Dr. George D. Snell (1903-1996). Dr. Snell received the Nobel Prize in 1980 for fundamental contributions to the field of immunogenetics. His pioneering work at The Jackson Laboratory provided the basis for understanding the graft rejection process and the development of human organ transplantation. Identification of individual genes contributing to the multifactorial inheritance of transplant tolerance was a formidable challenge in 1942, when only 8 linkage groups and 23 mouse loci had been identified [2]. Dr. Snell established the first localization of a histocompatibility gene, on what is now known as Chromosome (Chr) 17 [3]. The subsequent mapping of more than 40 histocompatibility loci has contributed significantly to the development of the mouse genetic map.

This brief review highlights a few of the more than 200 papers presented in Paris.

International Mouse Chromosome Committee meetings. Issues related to the generation of consensus genetic maps from multiple independent crosses and the development of new formats for presentation of genetic and physical data were discussed at this year's committee meetings. Software for on-line editing of the maps was introduced by The Jackson Laboratory informatics group. Henceforth, the Committee Maps will be continuously updated by committee members. The committee-generated consensus maps can be accessed electronically at (http://www.informatics.jax.org/ mgd.html). Mammalian Genome will continue to publish a printed version on an annual basis, with the next issue scheduled for publication in August 1997.

Mutant generation and identification. The discovery of the lysosomal trafficking regulator gene Lyst responsible for the mouse beige mutation and the human Chediak Higashi syndrome was described by Maria Barbosa (University of Florida) and Karen Moore (Millenium). The two groups initially identified nonoverlapping cDNAs that were later shown to be $5^{\prime}$ and $3^{\prime}$ portions of the large Lyst transcript [4,5]. Identification of the calcium channel alpha subunit gene responsible for the allelic neurological mutations tottering and leaner was reported by Colin Fletcher (Frederick Cancer Center, Md.). This work was facilitated by a congenic strain that narrowed the nonrecombinant interval and the availability of two independent alleles. From comparative mapping, Johan-

Correspondence to: M.H. Meisler nah Doyle (Oak Ridge National Laboratory) predicted that the same gene would be mutated in the human disorders Familial Hemiplegic Migraine and Episodic Ataxia 2, a prediction that was recently confirmed [6]. A defect in the major intrinsic protein gene Mip was shown to be associated with cataract development in the Hfi mouse by Duska Sidjamin (Univ. Pennsylvania). Jonathan Stoye (Mill Hill) described the cloning of the $F v l$ gene responsible for resistance to the murine leukemia virus (MLV). This locus encodes a product with homology to retroviral gag genes, suggesting that endogenous mammalian retroviruses may serve unsuspected biological functions.

A screening program for identification of mutations affecting vision and hearing was described by Muriel Davisson (Jackson Laboratory). Seventeen new vision and 15 new vestibular variants have already been identified. A Mutagenesis Handbook Database with protocols, screening techniques, and updates on projects in progress is under development at the MRC, Harwell (http:// www.mgu.har.mrc.ac.uk/MGU-welcome.html). Maya Bucan (Univ. Pennsylvania) described the screening of 1000 offspring of ENU mutagenized males, using a protocol that combines a whole genome screen for dominant behavioral traits with a hemizygous screen for novel mutations within the $\mathrm{W}^{19 \mathrm{H}}$ deletion on Chr 5 .

Physical and genetic maps. A genetically anchored YAC framework map of the mouse X Chr was described by Paul Denny (MRC, Harwell) and represents the first step towards a complete physical map of this $160-\mathrm{Mb}$ chromosome. Results of a large-scale sequence spanning $94 \mathrm{~kb}$ around the Xist locus were presented by Marie-Christine Simmler (Pasteur Institute), including identification of a unique transcript expressed in testis, and comparison of methods for sequence analysis. Substantial progress on the physical map and DNA sequencing of mouse Chr 16 was reported by Roger Reeves (Johns Hopkins, Baltimore, Md.). Sequence comparison with human $\mathrm{Chr} 21$ led to the identification of genes not recognized by computer software. Analysis of a 2-Mb contig of the $\mathrm{H} 2$ major histocompatibility region revealed an ancient family of eight MHC class I genes (Kirsten Fischer Lindahl, University of Texas Southwestern, Dallas).

Genetic mapping of expressed sequence tags in the mouse complements DNA sequence analysis and provides access to cDNA libraries from early developmental time points and diverse tissues. The chromosomal mapping of $>1000$ brain cDNAs using SSCP to detect interstrain variation was reported by David Beier (Harvard Medical School, Boston). Greg Lennon (Lawrence Livermore, California) described the mouse EST project of the I.M.A.G.E. consortium. Forty thousand cDNAs from 22 cDNA libraries, including normalized libraries prepared by Bento Soares, have been sequenced at Washington University, St. Louis and deposited into dbEST (http://www-bio.llnl.gov/bbrp/image/ image.html). The goal is to complete 400,000 sequences in 2 years, and the donation of additional mouse cDNA libraries for this project was requested. Systematic mapping of the mouse ESTs is not yet planned. The fact that $80 \%$ of the positionally cloned human disease genes are represented in the human EST database (51/62) 
indicates that completion of the mouse EST project will have a major impact on positional cloning.

New technology and resources. A novel two-step method for generating nested deletions around a locus was described by John Schimenti (Jackson Laboratory). After targeted integration of a negatively selectable marker in ES cells, cells are irradiated to generate random chromosomal deletions and grown in selective medium to isolate clones that have lost the marker. The length and extent of the resulting set of nested deletions can be determined by PCR assay for loss of heterozygosity, with ES cells derived from an $F_{1}$ hybrid between two inbred strains. The SHIRPA protocol for standardized evaluation of new mutants was described by J. E. Martin (Royal London Hospital). One person can evaluate 100 mice per day with 5-step protocol that includes assessment of body posture, spontaneous activity, transfer arousal, piloerection, startle response, gait, mobility, grip strength, pinna and corneal reflex, and response to toe pinch and handling. Adoption of a standardized protocol would provide objective, reproducible data and would facilitate comparison of mutant phenotypes described in different laboratories. CAP trapper, an efficient method for constructing full-length cDNA libraries on the basis of chemical introduction of a biotin group into the diol residue of the cap site followed by selection for full-length cDNA with streptavidin-coated magnetic beads, was described by P. Carninci (RIKEN, Japan).

Chromatin structure and gene regulation. On the basis of analysis of mild alleles at the Steel locus that are located at distances up to $180 \mathrm{~kb}$ from the MCGF structural gene, Neal Copeland (Frederick Cancer Center, Md.) proposed that long-distance position effects may be more common in the mammalian genome than has been recognized [7]. Bruce Cattanach (Harwell, UK) presented a mouse model for the Angelman and Prader Willi syndromes that affect imprinted loci; the relationship is supported by expression studies, comparative mapping, and phenotypic analysis. A new imprinted region of mouse Chr 2 was described by Jo Peters (Harwell, UK). Analysis of targeted mutations of Paxl by Rudi Balling (Munich) demonstrated that the severe spontaneous alleles of undulated that involve sizable deletions are likely to disrupt additional genes.

Rat and hamster genetic maps. A complete genetic map of the hamster genome was generated with the RLGS two-dimensional DNA technology by Yasushi Okazaki with Yoshihide Hayashizaki (RIKEN, Japan). The map was used to localize a cardiomyopathy locus and will facilitate genetic analysis of other hamster mutants [8]. Recent progress on the genetic map of the rat includes establishment of the database RATMAP (http://ratmap.gen.gu.se) and organization of a Rat Genetic Nomenclature Committee. Goran Levan (Goteborg University, Sweden) reported that 1600 genes have been mapped to rat chromosomes by linkage analysis and FISH, providing $85 \%$ coverage of the genome.

Informatics and databases. MRC Harwell's new web site (http:// www.mgu.har.mrc.ac.uk/MGU-welcome.html) will provide genetic imprinting maps, chromosomal aberrations, searchable lists of mouse frozen embryo and mutant stocks, and a mutagenesis handbook (Mark Strivens, MRC Harwell). Judith Blake and Janan Eppig (The Jackson Laboratory) described the evolving status of the Mouse Genome Database, a comprehensive database for genetic and biological data (http://www/informatics.jax.org), and the Gene Expression Database for Mouse Development (GXD) (http:/ /www.informatics.jax.org/gxd.html), a database containing images of embryonic expression data.

Quantitative trait analysis. Several groups reported progress in identification and refined localization of quantitative trait loci (QTLs). Phenotypes currently under investigation include obesity, diabetes, insulin resistance, hyperactivity in the rat, Alzheimer disease, brain and retinal development, antibody responsiveness, and psychomotor response to cocaine. Lorraine Flaherty (Albany, NY) identified loci affecting contextual memory in crosses between inbred strains and also in progeny of a mutagenesis experi- ment. Miroshi Masuya (Mishima, Japan) described two loci that modify the preaxial polydactyly phenotype of Rim4 mutant mice as well as several classic limb mutants, suggesting an important role in formation of the anterioposterior axis of the limb. Ed Wakeland (U. Florida, Gainesville) described the phenotypes of four congenic lines generated by marker-assisted selection to separate the components of susceptibility to systemic lupus erythematosus (SLE) in the NZM2410 mouse strain.

Guest lectures. Jean Weissenbach (Pasteur Institute) reported on the current status of the human genome project, including the mapping of more than 15,000 ESTs by a consortium of American and European laboratories [9]. The genetic length of the CEPHbased human genetic map is $3700 \mathrm{cM}$. Mutations in microsatellite markers were found to be responsible for some apparent double recombinants. The future role of silicon chip-based mutation detection for genetic disorders was also discussed. Bernard Dujon (Pasteur Institute) described the challenge of deriving functional information from the complete sequence of the yeast genome [10]. This genome contains approximately 6000 protein coding genes, only $1 / 3$ of which were recognized prior to the sequence analysis. The EUROFAN project, with 138 participating laboratories, will focus on functional analysis of the 2000 yeast genes whose function is currently unpredictable. Since a significant fraction of yeast genes have sequence similarity to mammalian genes, the functional genomics of the yeast will have profound implications for mammalian genetics. Richard Mural (Oak Ridge National Laboratory) described improvements in the GRAIL software for exon prediction, and demonstrated the powerful analytical and graphical programs now available for genomic sequence (11). He emphasized the importance of developing new methods of annotation that would enable investigators to contribute data from experimental analysis of gene function as additions to sequence entries in the databases.

Future meetings: The Eleventh Mouse Genome Conference, organized by Edward Wakeland (U. Florida, Gainesville), will be held from October 12 to 16 in St. Petersburg, Florida. Registration information is available electronically at the website (http:// mcbio.med.buffalo.edu/11imgc/) and by email (dmiller@mcbio. med.buffalo.edu). Membership in the International Mammalian Genome Society (IMGS), which sponsors these Conferences, is open to all interested investigators. Special membership rates are available for conference registration and subscriptions to Mammalian Genome. To become a member of the IMGS, contact Darla Miller, IMGS Administrative Manager, Department of Molecular Biology, Roswell Park Cancer Institute, Buffalo, New York 14263, USA.

Acknowledgments. This conference was funded by the Institut National de la Sante et de la Recherche Medicale (INSERM), the U. S. National Center for Human Genome Research (HG00756), and the U. S. Department of Energy. Support was also received from the International Mammalian Genome Society and from Mammalian Genome.

\section{References}

1. Avner P, Amar I, Dandolo L, Guénet J-L (1988) Genetic analysis of the mouse using interspecific crosses. Trends Genet 4, 18-23

2. Russell ES (1985) A history of mouse genetics. Annu Rev Genet 19, $1-28$

3. Gorer PA, Lyman S, Snell GD (1948) Studies on the genetic and antigenic basis of tumour transplantation. Linkage between histocompatibility gene and fused in mice. Proc R Soc London Ser B 135, 499-505

4. Barbosa MDFS, Nguyen QA, Tchernev JA, Ashley JA, Detter JC, Blaydes SM, Brandt SJ, Chotai D, Hodgman C, Solari RCE, Lovett M, Kingsmore SF (1996) Identification of the homologous beige and Chediak-Higashi syndrome genes. Nature 382, 262-265

5. Perou CM, Moore KJ, Nagle DL, Misumi DJ, Woolf EA, McGrail SH, Holmgren L, Brody TH, Dussault BJ, Jr, Monroe CA, Duyk GM, 
Pryor RJ, Li L, Justice MJ, Kaplan J (1996) Identification of the murine beige gene by YAC complementation and positional cloning. Nature Genet 13, 303-308

6. Ophoff RA, Terwindt GM, Vergouwe MN, van Eijk R, et al. (1996) Familial hemiplegic migraine and episodic ataxia type- 2 are caused by mutations in the $\mathrm{Ca}^{2+}$ channel gene CACNL1A4. Cell 87, 543-552

7. Bedell MA, Jenkins NA, Copeland NG (1996) Good genes in bad neighborhoods. Nature Genet 12, 229-232

8. Okazaki Y, Okuizumi H, Ohsumi T, Nomura O, Takada S, Kamiya M, Sasaki N, Matsuda Y, Nishimura M, Tagaya O, Muramatsu M, Hay- ashizaki Y (1996) Genetic-linkage map of the syrian hamster and localization of cardiomyopathy locus on chromosome 9QA2.1-B1 using RLGS spot-mapping. Nature Genet 13, 87-90

9. Weissenbach J (1996) Landing on the human genome. Science 274, 2055-2070

10. Dujon B (1996) The yeast genome project—what did we learn? Trends Genet 12, 263-270

11. Uberbacher EC, Xu Y, RJ Mural (1996) Discovering and understanding genes in human DNA sequence using GRAIL. Methods Enzymol 266, 259-281 